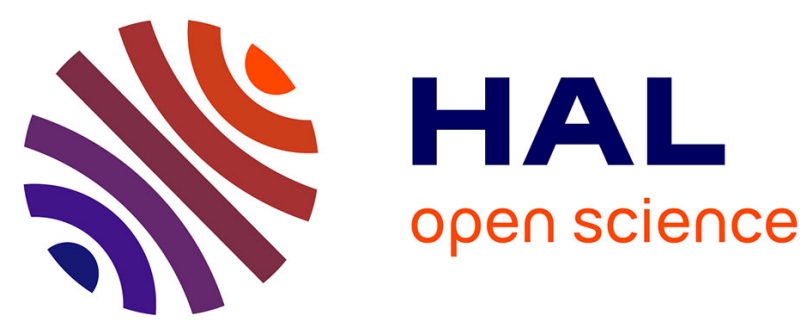

\title{
OPTICAL QUALITY OF TILTED SPHERICAL MIRROR UNSTABLE RESONATORS
}

\author{
C. Cason, R. Jones, J. Perkins
}

\section{To cite this version:}

C. Cason, R. Jones, J. Perkins. OPTICAL QUALITY OF TILTED SPHERICAL MIRROR UNSTABLE RESONATORS. Journal de Physique Colloques, 1980, 41 (C9), pp.C9-385-C9-391. 10.1051/jphyscol:1980952 . jpa-00220606

\section{HAL Id: jpa-00220606 https://hal.science/jpa-00220606}

Submitted on 1 Jan 1980

HAL is a multi-disciplinary open access archive for the deposit and dissemination of scientific research documents, whether they are published or not. The documents may come from teaching and research institutions in France or abroad, or from public or private research centers.
L'archive ouverte pluridisciplinaire HAL, est destinée au dépôt et à la diffusion de documents scientifiques de niveau recherche, publiés ou non, émanant des établissements d'enseignement et de recherche français ou étrangers, des laboratoires publics ou privés. 


\title{
OPTICAL QUALITY OF TILTED SPHERICAL MIRROR UNSTABLE RESONATORS
}

\author{
C.C. Cason, R.W. Jones and J.F. Perkins*
}

* Army Directed Energy Directorate, U.S. Army Missile Comand, Redstone Arsenal, AZabama 35809, U.S.A.. * University of Alabama in Huntsvilie, Huntsville, Alabama 35804, U.S.A..

\begin{abstract}
Résumê.- Les lasers chimiques de grande puissance ont souvent des régions de gain de grand allongement, pour lesquelles un rësonateur instable avec différentes amplifications dans les deux dimensions transversales est désirable. Ce papier considère une nouvelle classe de résonateurs instables qui rêalisent la propriêté d'amplifications différentielles avec des miroirs sphériques en inclinant convenablement les miroirs au moyen de grands angles. La qualité du rendement optique a été recherchée théoriquement et expérimentalement. L'on a découvert que des résonateurs instables, produisant des faisceaux lasers presque uniphases, peuvent être fabriqués en utilisant cette nouvelle approche. Une propriété spéciale permet aux amplifications transversales d'être continuellement modifiées, ce qui est une aide importante pour la dynamique des gaz de grande puissance ou pour les activités de dêveloppement expérimental des lasers chimiques quand la longueur de la trajectoire de l'accroissement du flux est modifiée.
\end{abstract}

\begin{abstract}
High power chemical lasers often have high aspect ratio gain regions, for which an unstable resonator with different magnifications in the two transverse dimensions is desirable. This paper considers a new class of unstable resonators which achieves the differential magnification property with spherical mirrors by suitably tilting the mirrors through large angles. Output optical quality has been investigated theoretically and experimentally. It was found that unstable resonators producing near uniphase laser beams can be fabricated using this new approach. A special property allows the transverse magnifications to be continuously varied, a significant aid for high power gas dynamic or chemical laser experimental development activities when the flow gain path length is varied.
\end{abstract}

\section{INTRODUCTION}

In various applications, including in particular high power gas dynamic and chemical lasers with relatively short gain lengths, compared to the gain height there is a need for unstable laser resonators which produce a collimated output beam but have asymmetric magnification, i.e. $M_{x} \neq M_{y}$. Such resonators have been achieved by others by use of mirrors with toroidal figuring, but there are substantial difficulties involved in fabrication and alignment of such mirrors. Special cases of the toroidal resonator include the cylindrical mirror system. Wave optics analyses of these special cases show that mode control difficulties exist. Fabrication of toroidal mirrors does not permit the control of the surface radii, $R_{x}$ and $R_{y}$ of each mirror to much better than about $2 \%$. This does not always allow optimum mirror separations to provide a uniphase beam in both transverse dimensions simultaneously. Additionally some transverse mode control is sacrificed in the toroidal resonator concept. High energy laser research activities will require several sets of these complicated toroidal resonator designs, as the gain in the flow direction is varied. A need has evolved to develop a resonator that has the simultaneous properties of producing a nearly uniphase beam, of allowing a continuously variable magnification in the two orthogonal directions and of accommodating a continuously variable aspect ratio gain medium. We have previously [1] proposed a new class of resonators which achieves all these properties without the dis- 
advantages of nonspherical mirror figure requirements. The designs involve tilting both resonator spherical mirrors by rather large angles. The present design concept is applicable to either standing-wave or ring resonators. [2,3]

The purpose of this paper is to report on a recent investigation of this new resonator to determine its usefulness. The approach is based on an analysis of the large degree of astigmatism introduced by the tilt of the convex mirror and predicting its cancellation by suitable choice of mirror spacing and tilt of the concave mirror. In the previous work we have given the design equations and have confirmed the symmetric magnification property. It was concluded that the tilted-spherical-mirror resonator scheme offers considerable practical advantages over other schemes for achieving asymmetric magnification, but the critical problem of residual aberrations introducing deterioration of output optical quality needed further investigation. The present report describes a combined theoretical-computational study of beamquality properties of tilted-spherical-mirror resonator concepts that was compared to results of laboratory experiments.

\section{THEORETICAL DEVELOPMENT}

This study considers the effect of one complete passage from convex to concave mirror on an initially collimated beam, which is essentially a ring-resonator configuration. Both cases of relative sign of spherical-mirror tilt angles are considered; these are referred to as $U$ and $Z$ configurations, as illustrated in Figure 1. It is assumed throughout that coordinate systems are chosen such that the $\mathrm{x}$-axis is parallel to the axis about which mirrors are tilted. The z-axis is along the nominal propagation direction in any region.

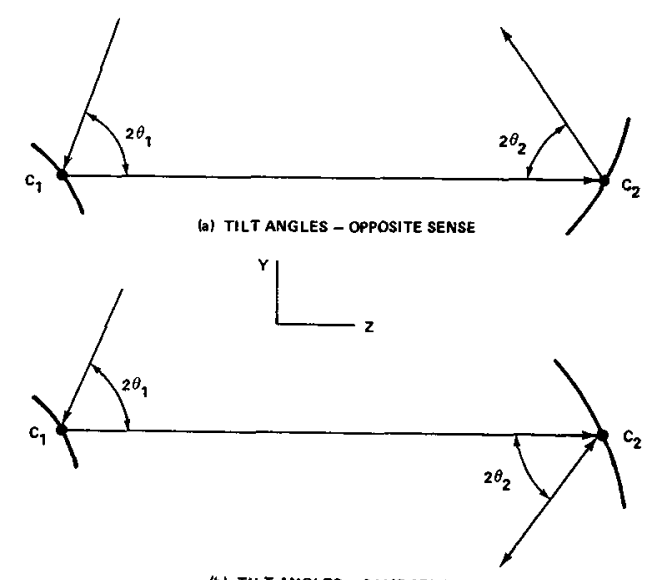

(b) TILT ANGLES - SAME SENSE

Figure 1. Schematic drawing of tilted-mirror arrangements of U-type (top drawing) and Z-type (bottom drawing).

A two-dimensional approximate treatment was first pursued theoretically. That is, attention is confined to a plane perpendicular to the mirror-tilt axis. From the closed-form analysis one can obtain predictions of the magnitude of one of the major contributors to residual aberrations, namely the $\mathrm{y}^{3}$ term. For brevity, the derivation is omitted and only the final result is quoted. We obtained

$$
z=\frac{1}{R_{2}^{3} \cos ^{3} \theta_{2}}\left[-\left|R_{1}\right| \sin \theta_{1} \pm R_{2} \sin \theta_{2}\right] y^{3}
$$

Usually values of $R_{1}, R_{2}$, and $\theta_{1}$ are given. The value of $\theta_{2}$ can then be determined by the basic design equations. [1] In the above equation for the $y^{3}$ aberration the upper sign on the second term in square brackets corresponds to a $\mathrm{Z}$ configuration, while the lower sign corresponds to a U configuration. Thus the $\mathrm{Z}$ configuration is preferred over the $U$ configuration as regards aberrations.

Deterioration of the optical quality of the output beam is essentially determined by the root-meansquare (rms) value of Optical Path Difference (OPD), $z$, measured in units of wavelength, relative to a suitable reference plane for a collimated output 
beam. The rms variations are considerably smaller than peak-to-peak or center-to-peak variations, and the choice of reference plane is important. The rms value for an untilted reference plane is:

$$
\mathrm{z}_{\text {rms, nt }}=0.378 \mathrm{z}_{\mathrm{cp}} \text {. }
$$

The rms value of the OPD after choosing an optimal tilt for the reference plane can then be shown to be:

$$
z_{\text {rms, opt }}=0.151 \mathrm{z}_{\mathrm{cp}} \text {. }
$$

Some numerical examples are given in Table I

which is representative of chemical-1aser resonator designs.

Table I. Two-Dimensional Approximate OPD Predictions (in Microns) for a $Z$ Configuration Trave1ling-Wave Resonator with $R_{1}=-1481 \mathrm{~cm}, \mathrm{R}_{2}=$ $3703 \mathrm{~cm}, \mathrm{Y}=\mathrm{y}=7.5 \mathrm{~cm}$, for Various Values of Convex-Mimiror Tilt Angle $\theta_{1}$

\begin{tabular}{|c|c|c|c|}
\hline$\theta_{1}(\mathrm{deg})$ & $z_{\mathrm{cp}}$ & $z_{\mathrm{rms}, \mathrm{nt}}$ & $z_{\mathrm{rms}, \mathrm{opt}}$ \\
\hline 10 & 0.013 & 0.005 & 0.002 \\
20 & 0.028 & 0.010 & 0.004 \\
30 & 0.048 & 0.018 & 0.007 \\
45 & 0.100 & 0.038 & 0.015 \\
50 & 0.130 & 0.049 & 0.020 \\
55 & 0.174 & 0.066 & 0.026 \\
60 & 0.240 & 0.091 & 0.036 \\
65 & 0.352 & 0.133 & 0.053 \\
68.6 & 0.491 & 0.185 & 0.074 \\
\hline
\end{tabular}

From these estimates, the optical quality should be quite acceptable at infrared wavelengths, when rms and reference-plane-tilt effects are accounted for, except for the rather extreme case of the mirrors being used for the interferometric testing.

Such two-dimensional estimates would be considered quite uncertain if standing alone. Results of three-dimensional ray-tracing calculations indicate that the two dimensional estimates give useful semiquantitative guidance to the expected degree of optical quality degradation for an ideally aligned system. On the other hand, the two-dimensional considerations give no indication whatever of the sensitivity of optical quality to various systems parameters, including increments to mirror separation $\mathrm{I}$, and concavemirror-tilt-angle $\theta_{2}$ for a specified value of $\theta_{1}$. Also the two-dimensional results give no information about expected shapes of interferograms.

The major portion of the theoretical-computational part of this study has been concerned with developing and applying three-dimensional ray-tracing computational methods and with correlating the results (primarily in the form of quasi-interferograms) with the experimental interferograms. The pair of mirrors used for comparisons between experimental and computational results had radii of curvature of $R_{1}=-290 \mathrm{~cm}$, $\mathrm{R}_{2}=675 \mathrm{~cm}$. These were deliberately chosen to give rather large aberrations in order to facilitate the comparisons.

Equations employed in ray tracing assume a collimated beam incident on the convex mirror. Given $x$ and $y$ coordinates of an incident ray measured relative to the central ray, the computer program calculates the path of the ray through the system and determines (a) direction cosines after reflection from the concave mirror, measured in a coordinate system with the positive z-axis parallel to the central ray and (b) the OPD between the ray considered and the central ray. The OPDs are of primary interest, and are calculated for a position close to the concave mirror. There is a good deal of order in the calculated OPDs; their functional dependence on $\mathrm{x}$ and $\mathrm{y}$ can be well represented by several terms of a Taylor's expansion. Terms $\mathrm{C}_{03} \mathrm{y}^{3}$ and $\mathrm{C}_{21} \mathrm{x}^{2} \mathrm{y}$ are of primary import ance.

The experimental data regarding optical quality from tilted spherical mirror resonators are in the form of interferograms. While providing very precise and detailed information about the optical systems, 
inteferograms do not immediately (without an intervening measurement and data-reduction process) lead to quantities predicted by the calculations. Therefore, it seemed useful to convert the calculated results into the form of computer-simulated quasi-interferograms, thereby facilitating visual comparison of experimental and computational results. One can obtain quantitative measures of optical quality from the computational results after confirming them by comparison with experimental interferograms. For this purpose the computer generates a fairly large array of OPDs obtained by evaluation of the truncated series expansion. The array of numerical values is converted to an array of alphabetic characters and blanks and used to generate a one-page printer plot whose general appearance simulates that of an interferogram.

Three-dimensional calculations were carried out for the pair of mirrors used in the expeximental studies, using several values of the convex-mirror tilt angle $\theta_{I}$. The three-dimensional predictions of the optimized rms OPD range from some $25 \%$ to $50 \%$ larger than the corresponding two-dimensional predictions for these cases.

\section{EXPERIMENTAL AND THEORETICAL RESULTS}

The results of experiments agree satisfactorily with the computational results obtained in the three-dimensional calculations of wavefront OPDs. The set of mirrors was chosen to give a moderate number of fringes across the output aperture which could be compared directly to calculated quasi-interferograms. This test represented an extreme case compared to the aberrations which would be present in the proposed high aspect ratio resonator mirrors but provided a direct correlation between theory and experiment.

Two types of resonator concepts were tested, the
$Z$ configuration and the $U$ configuration. Most of the interferograms were taken with the $\mathrm{Z}$ configuration experimental setup. Schematics of the experimental setup for the $Z$ and $U$ configurations are shown in Figure 2. A plane wavefront is produced by passing a helium-neon laser beam through a spatial filter (microscope objective plus pinhole) and collimating the resultant spherically divergent wavefront with a high quality objective lens of 6-in. diameter. This plane wavefront is then split into two components with a glass beamsplitter. One component (reference beam) is reflected directly into a focusing lers to a camera. The other portion (test beam) passes through the beansplitter to the convex resonator mirror, reflects to the concave resonator mirror, and is directed back through the beamsplitter into the camera by means of appropriately positioned mirror flats.

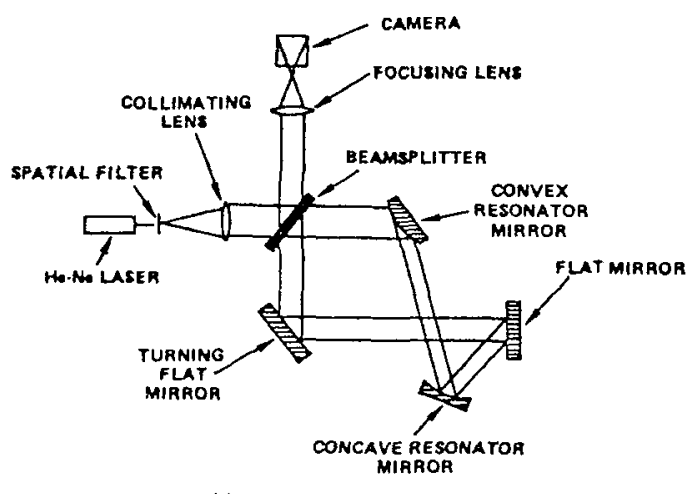

(b) Z CONFIGURATION

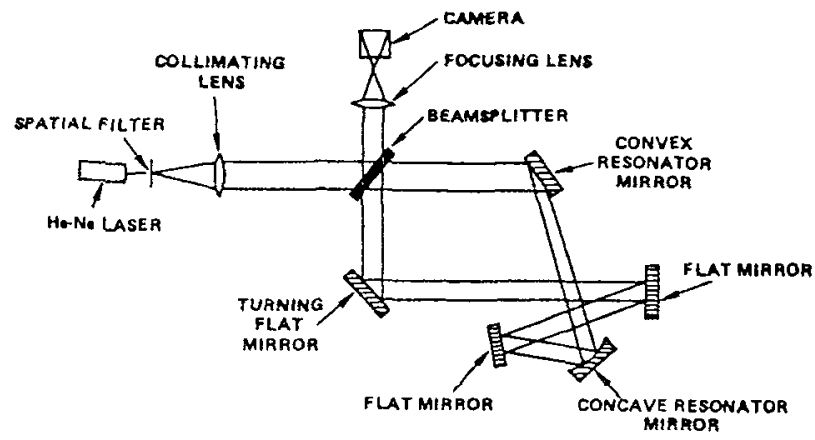

(b) U CONFIGUAATION

Figure 2. Experimental Arrangements for Obtaining Interferograms 

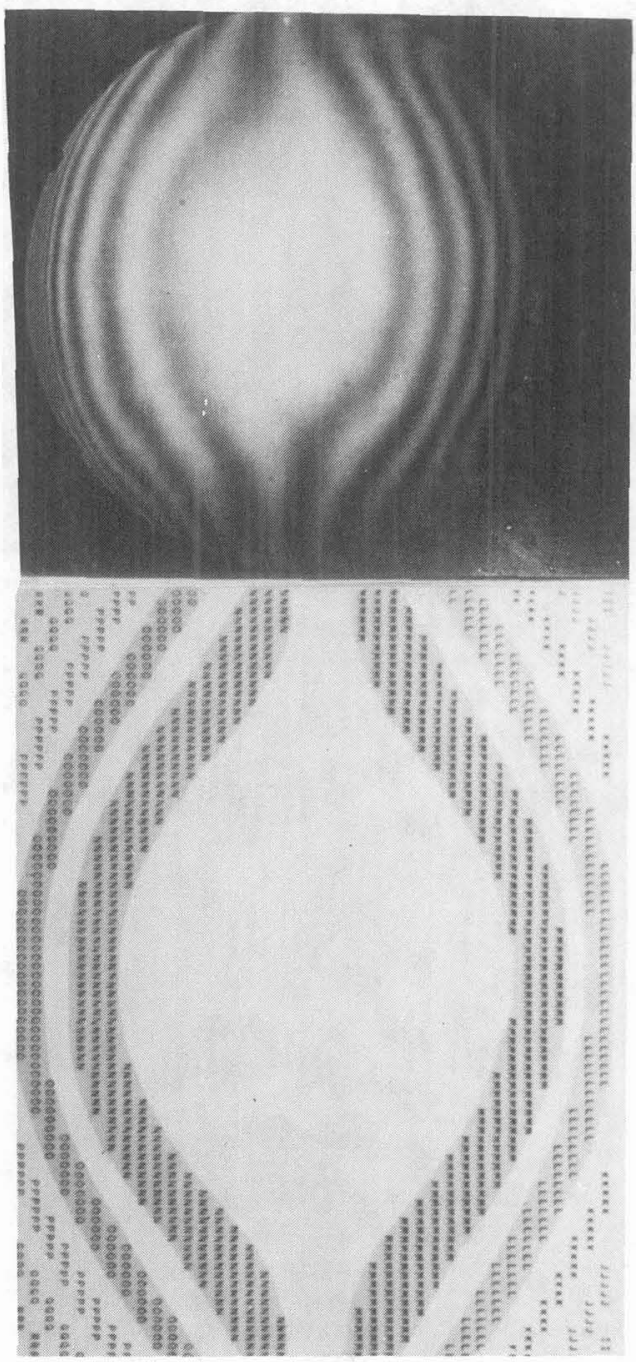

Fígure 3. 2 Configuration, $\theta_{1}=45^{\circ}$, $\mathrm{RBT}=-0.074$

The interferograms were obtained by superimposing the focal points of the two beams from the beamsplitter (by tilting the turning flat). There is a certain arbitrariness in this adjustment - the interferogram obtained depends strongly upon the angle between the reference beam and the test beam (wavefront tilt). This variability made it difficult to compare exactly the quasi-interferograms obtained analytically with the experimental interferograms.

Some examples are given in Figures $3-8$ of the comparison of experimental interferograms and the
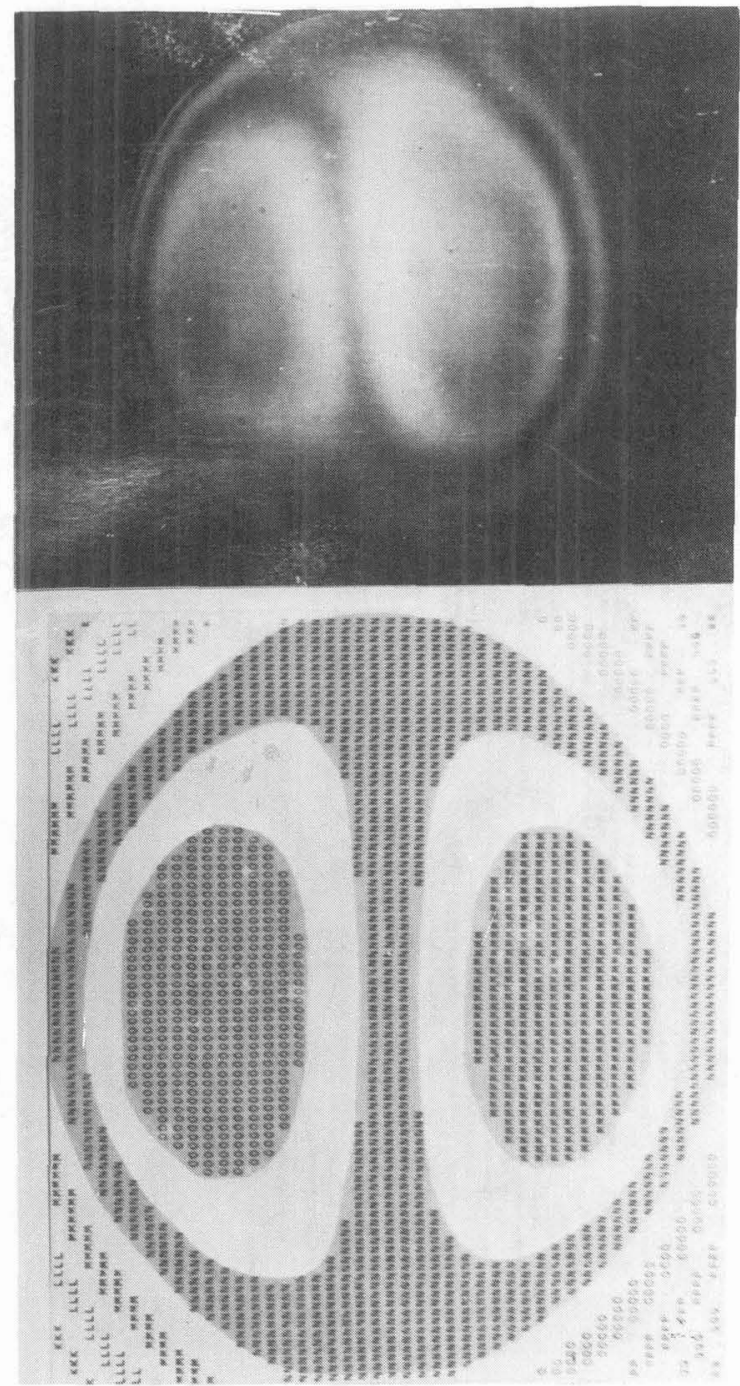

Figure 4. Z Configuration, $\theta_{1}=45^{\circ}$, RBT $=-0.296$

(approximately as judged by visual inspection) corresponding computer-generated quasi-interferograms. For each example, the experimental interferogram is shown in the top figure, while the computed quasi-interferogram is shown at the bottom. The agreement between the two is acceptable. The relative beam tilt, RBT, in units of $10^{-4}$ radians, is the angle between the reference plane and a plane perpendicular to the nomina1 propagation direction. The shape of interferograms changes in a systematic way as RBT is varied, as would be expected. A numerical value of RBT is 

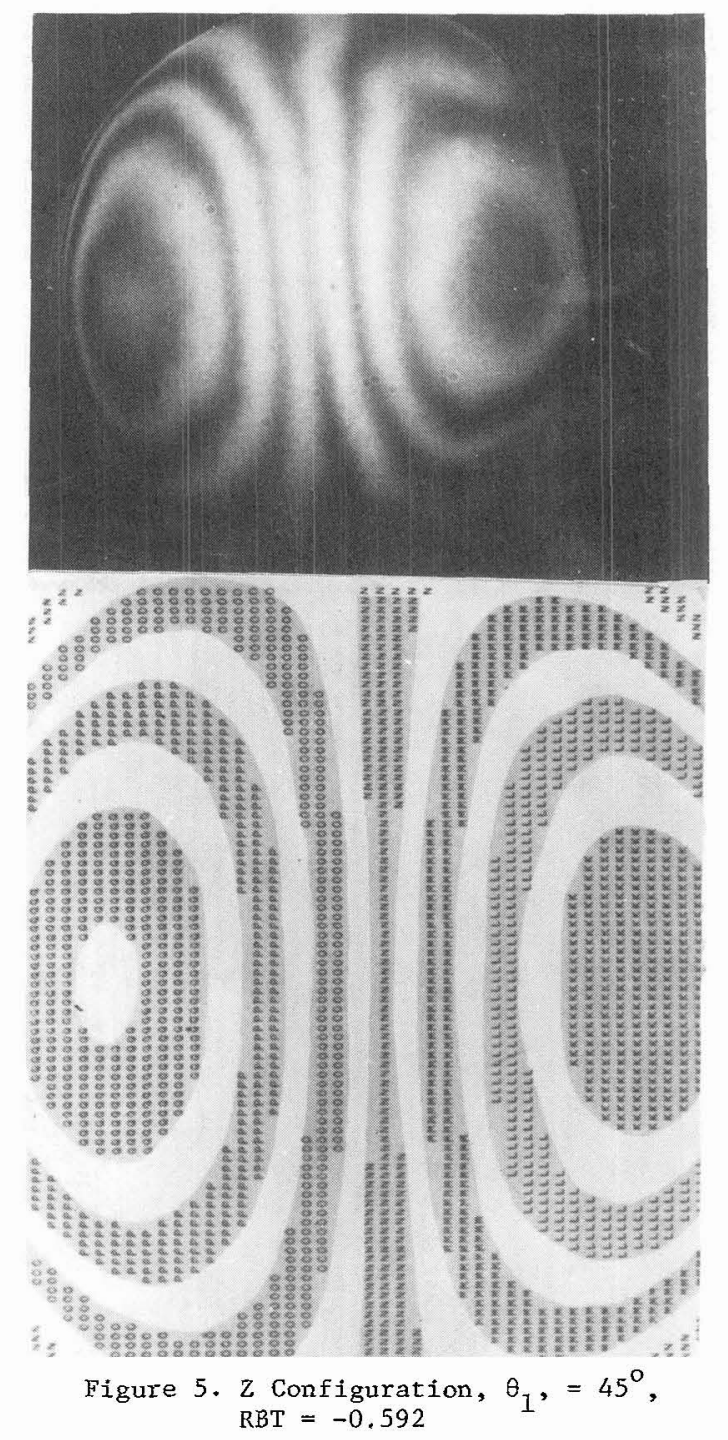

available only from the ray-tracing results. One can rather directly observe the change in interferogram shape with beam tilt in the experimental arrangement, however, and the change is of the same sort as found computationally. With increasing reference-beam tilt the pattern changes from a single oval to a double oval, as can be seen in Figures 3-5, for which $\theta_{1}=$ $45^{\circ}$.

Figure 6 shows the effect of a 7-milliradian misadjustment of the tilt angle ${ }_{2}$ of the concave mirror, for $\theta_{1}=45^{\circ}$.

Figure 7 shows a double-oval pattern for $\theta_{1}=70^{\circ}$,

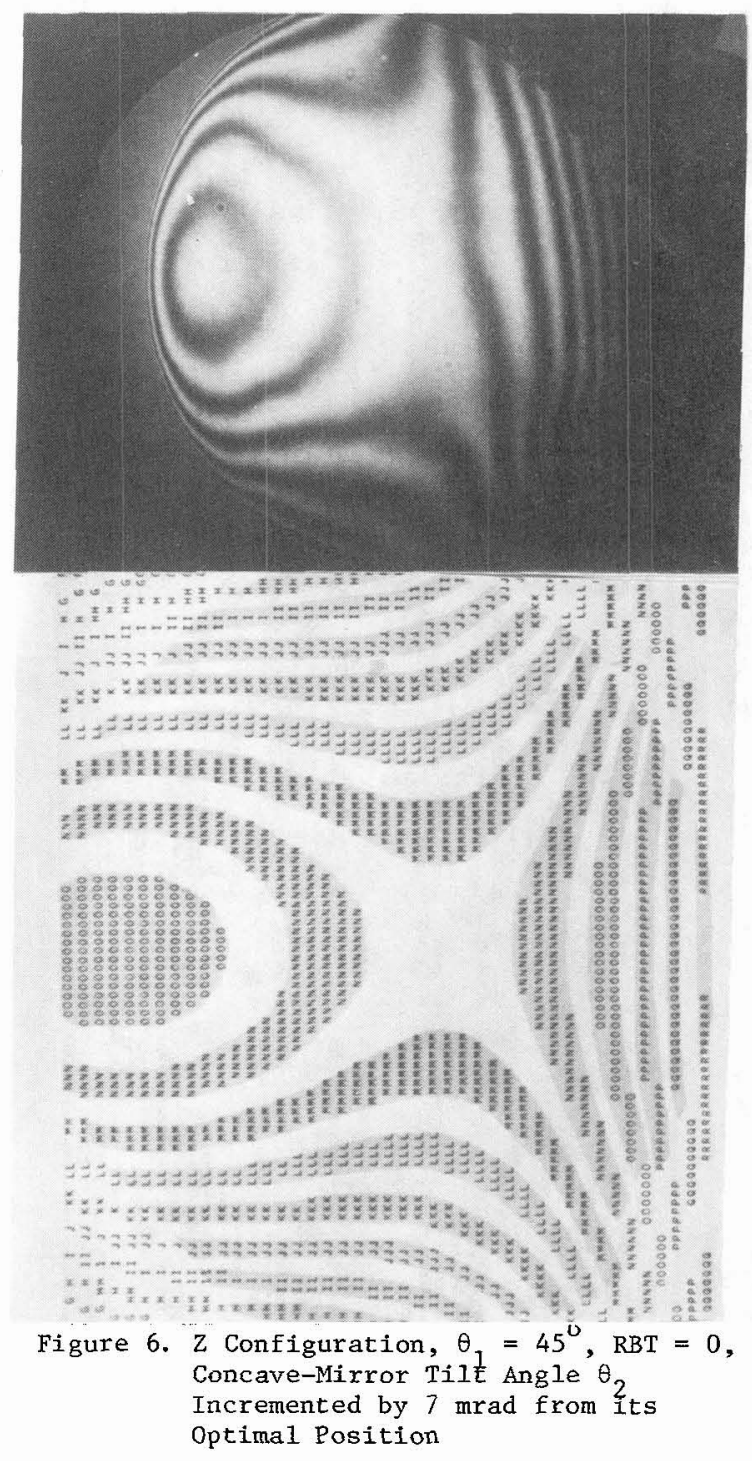

and is somewhat similar to the patterns seen in Figures 4 and 5 .

Figure 8 shows an interference pattern for a U-type configuration, which is qualitatively somewhat different than that of $\mathrm{Z}$ configurations.

SUMMARY AND CONCLUSIONS

The tilted spherical mirror resonator concept was validated theoretically and experimentally by choosing a convex/concave mirror set with small radii of curvature (small $\mathrm{f}$ number), The deliberately exaggerated aberrations were compared by experimental interferometry and computational plots of optical path 

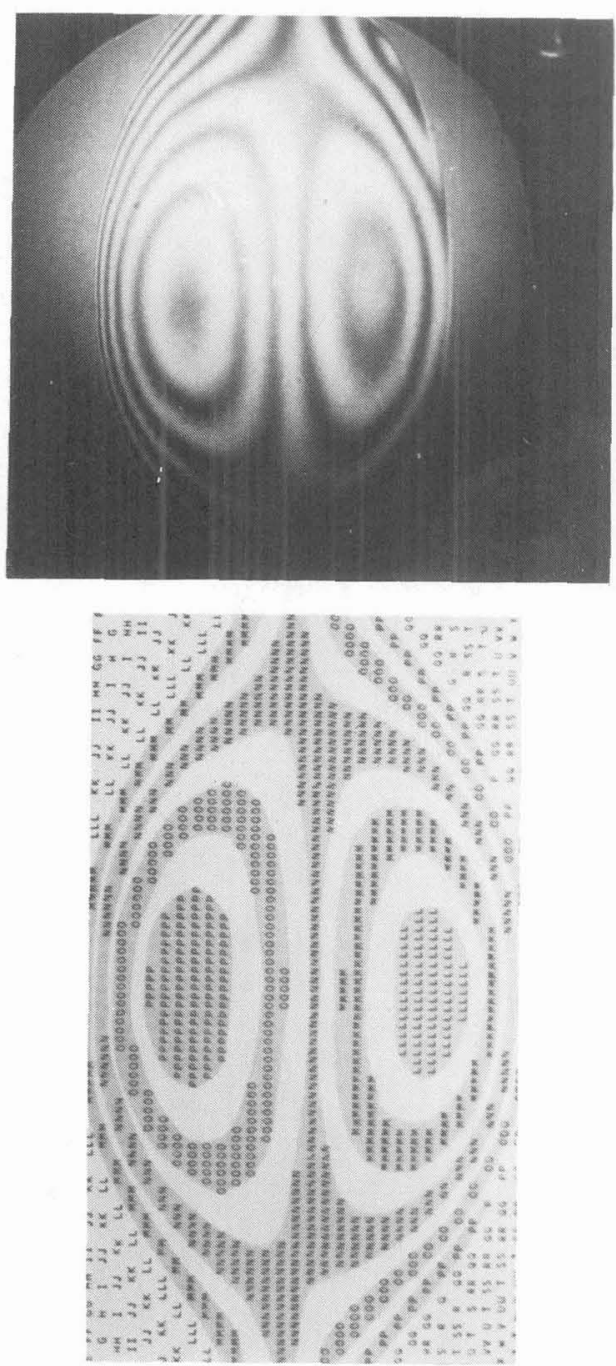
Figure 7. Z Configuration, $\theta_{1}=70^{\circ}$,
RBT $=-0.799$

differences. The results of experiment and theory agreed quantitatively.

The following conclusions were drawn from this work:

(1) The sense of relative tilt of the spherical mirrors affects the aberrations, the aberrations being smaller for the $\mathrm{Z}$ configuration.

(2) The dominant terms contributing to aberrations are of the form $y^{3}$ and $x^{2} y$ with $y^{3}$ predominating for high aspect ratio resonators.

(3) The concept has many advantages over toroidal resonators used with linear chemical lasers. Chemical
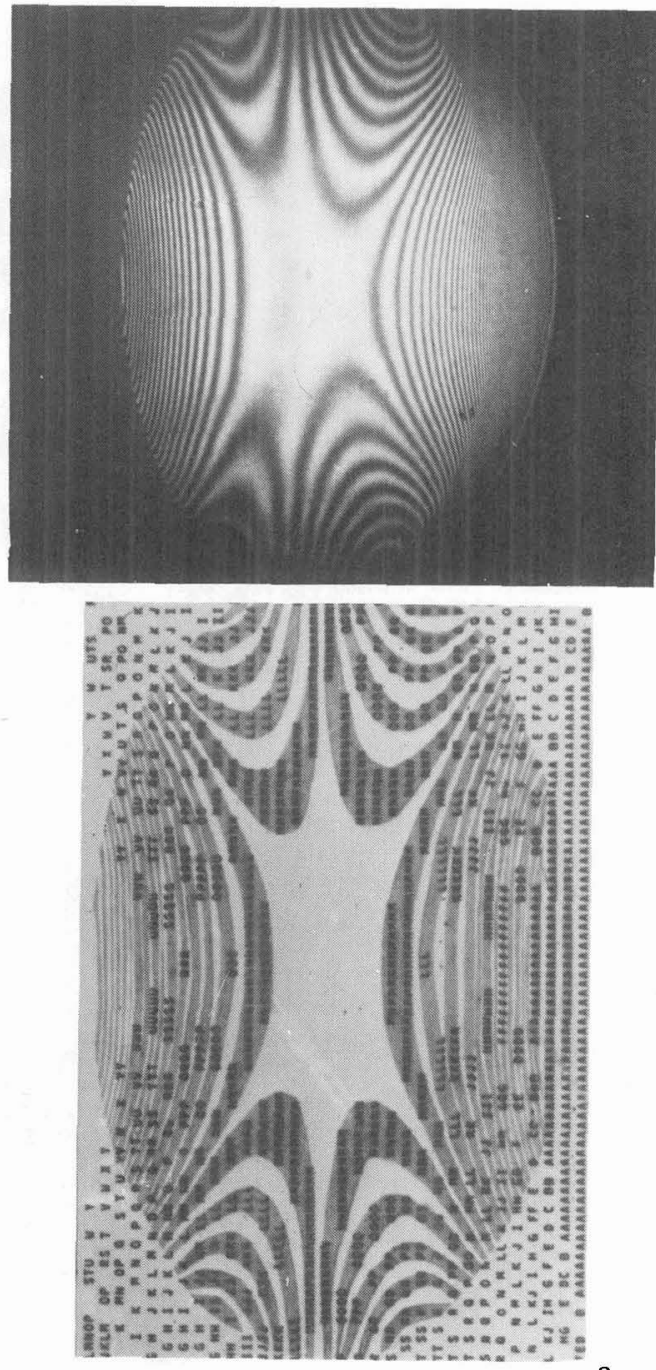
Eigure 8. U Configuration, $\theta_{1}=70^{\circ}$,
RBT $=0$

laser development programs experiment with varying values of $x_{c}$, thereby requiring several sets of toroidal mirrors for beam extraction. The present concept has the ability to vary $M_{x}$ continuously to account for planned variations in $x_{c}$ of interest in chemical laser development experiments, thereby requiring no more than one resonator.

\section{REFERENCES}

1. Charles Cason, R. W. Jones, and J. F. Perkins, Optics Letters 2, 145 (1978).

2. A. E. Siegman, App 1, Opt. 13, 353 (1974).

3. R. J. Freiberg, P. P. Chenausky, and C. J. Buczek, Appl. Opt. 12, 1140 (1973). 